\title{
FLUID-STRUCTURE INTERACTION ANALYSIS OF MARTIAN EXPLORATION PARAFOIL WITH DEPLOYABLE STRUCTURE BY COUPLING LIBRARY PRECICE
}

\author{
K. ISHIDA ${ }^{1}$, J. TOBARI ${ }^{2}$, Y. TAKAHASHI ${ }^{3}$, N. OSHIMA ${ }^{4}$, R. SHIBATA ${ }^{5}$ \\ ${ }^{1}$ Hokkaido University \\ Kita 13 Nishi 8, Kita-ku, Sapporo, Hokkaido 060-8628, Japan \\ k_ishida_k46@eis.hokudai.ac.jp \\ ${ }^{2}$ Hokkaido University \\ Kita 13 Nishi 8, Kita-ku, Sapporo, Hokkaido 060-8628, Japan \\ junki-david10@eis.hokudai.ac.jp \\ ${ }^{3}$ Hokkaido University \\ Kita 13 Nishi 8, Kita-ku, Sapporo, Hokkaido 060-8628, Japan \\ ytakahashi@eng.hokudai.ac.jp \\ ${ }^{4}$ Hokkaido University \\ Kita 13 Nishi 8, Kita-ku, Sapporo, Hokkaido 060-8628, Japan \\ oshima@eng.hokudai.ac.jp \\ ${ }^{5}$ National Institute of Technology Gifu College \\ 2236-2 Kamimakuwa, Motosu, Gifu 501-0495, Japan \\ ryos@gifu-nct.ac.jp
}

Key words: Inflatable Structure, Fluid-Structure Interaction, Coupled Problem, preCICE.

\begin{abstract}
Recently, the demand for planetary exploration has been increasing steadily owing to the development of space transportation systems. A parafoil technology for Mars exploration was developed by JAXA, in collaboration with several universities in Japan. This type of parafoil has no ram-air inlet because it is required for flights in low-density environments where dynamic pressure is insufficient. The parafoil primarily comprises flexible structures with optimal storage efficiencies, light weights, and high lift-drag ratios.

Parafoil technology can provide space explorations with several benefits, for example, the wide-range scanning of the Martian surface and atmosphere. However, parafoils are deformed during planetary exploration flights owing to aerodynamic forces, which can trigger aerodynamic instability. Although instability has been reported in wind tunnel experiments and flight tests, the detailed mechanism of this possible instability has not been clarified. Therefore, it is necessary to elucidate the detailed instability mechanism by numerical analysis, as it cannot be realized experimentally. In addition, it is necessary to introduce
\end{abstract}


fluid-structure interaction (FSI) analysis for flexible structures using a coupled method. The precise code interaction coupling environment (preCICE) coupling library is a powerful tool for the coupling analysis of fluid and structure solvers.

To evaluate the effect of coupling two physical fields, the analyses of the fluid and structure were conducted separately.

The results of the analysis verified that the wing deformed under a fluid force, which indicates the effectiveness of the FSI analysis model developed. A comparison with the single-field analysis demonstrated that the structure-derived frequencies in the FSI analysis appeared in the wing surface deformation and aerodynamic forces. However, the aerodynamic coefficients obtained by the FSI analysis converged to the same values as those obtained by the single fluid analysis, thus indicating that from a macroscopic perspective, structural deformation negligibly influences aerodynamic forces. Therefore, it is necessary to analyze a shape that is closer to the actual machine.

\section{INTRODUCTION}

Mars exploration missions have been widely conducted by several research institutes and universities. In Japan, JAXA, in collaboration with several universities, proposed a novel flight exploration technology in the Martian atmosphere. Free flights in the Martian atmosphere will enable us to explore wider areas at a distance closer to the ground than orbiters, as well as drop landers from the Martian sky to explore canyons and vertical holes that cannot be reached by conventional rovers. Therefore, flight exploration can provide higher freedom for Mars Although airplanes Martian atmosphere, Consequently, this pose Mars. Although increasing the wing area is an
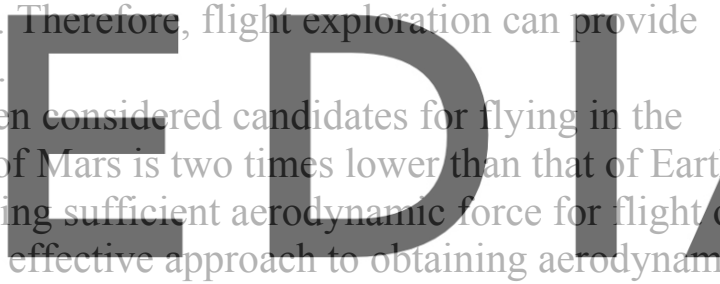
force, this increases the volume and weight of the spacecraft transported to Mars. In general

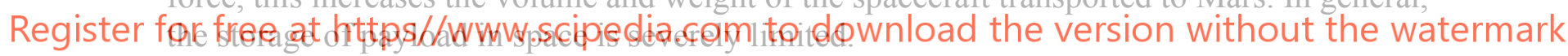

Therefore, Mars exploration parafoil has been suggested as a novel approach to flight

exploration [1]. Parafoil can consist of flexible and lightweight materials. It is considered beneficial owing to its optimal storage efficiency in limited spaces, irrespective of its large wing area. Paraglider parafoils, widely used in sky sports, etc., are air-type parafoils, which are deployed by passing air through openings in the leading edge of the wing. However, this type of parafoil is limited by its poor lift-drag ratio owing to the enormous drag generated by the air inlet at the leading edge. In addition, parafoils cannot be fully deployed in low-pressure environments [2].

First, we developed a fully sealed parafoil, which has a simple shape with its ram-air inlet blocked off. However, this parafoil was considered unsuitable for Mars exploration because it required a large amount of gas, and if a portion of its surface is damaged, the parafoil would lose its wing shape and ability to maintain airtightness.

A partially sealed parafoil, was developed to address this challenge, as illustrated in Figure 1 [3]. Unlike the fully sealed parafoil, only the inflatable girders inside the wing were fully sealed. The rib of the wing is made of balsa wood, and a thin polyethylene film was attached between the structural elements consisting of inflatable girders and ribs, forming an airfoil 
wing. This parafoil-type is advantageous because it requires a lesser amount of gas for deployment than the conventional type. It is also more reliable than the conventional type because the surface film does not need to be constantly airtight, whereas the fully sealed type can lose its functionality if only a part of the surface is damaged. Because the ribs are made of balsa wood, it is possible to construct an umbrella-like body without compromising the wing shape, although this would reduce its storage capacity.

In 2018, a wind tunnel test was conducted using JAXA's $6.5 \mathrm{~m} \times 5.5 \mathrm{~m}$ low-speed wind tunnel to determine the aerodynamic characteristics of the Mars exploration parafoil. This experiment demonstrated that the partially sealed parafoil tested had a high lift-drag ratio in the range of 5-6. However, when flow speed increased, the parafoil exhibited attitude instability in the roll direction. In 2018, a drop test was conducted using a multicopter for free flight. In this test, the parafoil was reversed after wing deployment [4]. Previous studies have pointed out that partially sealed parafoils exhibit less aerodynamic stability. Because the parafoil comprises a flexible structure, it has been confirmed that it deforms under fluid force. The experimental results obtained suggest that this deformation probably contributes to its aerodynamic instability; however, further details on the aerodynamic mechanism of parafoil's attitude instability of the parafoil are insufficiently studied. Considering these factors, FSI analysis is required to evaluate the influence of wing deformation on aerodynamic forces.

To conduct FSI analysis, it is necessary to select a suitable type of coupler. In this study, we adopted the precise code interaction coupling environment (preCICE) library as a coupling library based on [5], which comprehensively describes existing couplers, as well as the
advantages of the preCICE library. To date, several couplers, including preCICE, have been
developed. FSI*ce [5], which was developed for dedicated couplings of FSI analysis, controls
two types of physical fields: fluid and stricturd. MnCCI [6], which is a commercial softwdre,
is a relatively standard tool. In adidition, we adopted EMPIRE [5], which is an open sourde
software(OSS), that relies on the client-server approach. We implemented intercode communication using MPI, as well as sophisticated data mapping options for non-matching

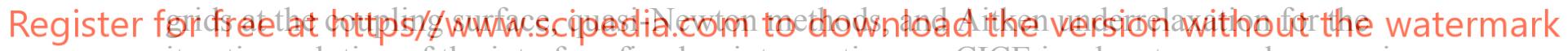
iterative solution of the interface fixed-point equation. preCICE is advantageous because in addition to FSI analysis, it works with virtually any solver. Additionally, it can be easily used for Multiphysics simulations as an OSS, and for maintaining communications with a remote supercomputer using the TCP/IP protocol. Because preCICE is based on a partitioned coupling approach, existing sophisticated solvers with established discretization methods can be used for both fluid and structural analyses. Furthermore, adapters are provided to OSS solvers.

Therefore, we selected preCICE for convenience, and the purpose of this study was to develop an FSI analysis model to investigate the aerodynamic behavior of a deployable flexible structure. 


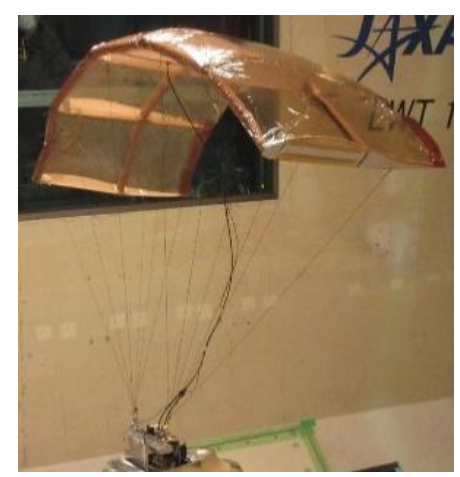

Figure 1: Partially sealed parafoil

\section{NUMERICAL METHODS}

\subsection{Fluid analysis}

The present flow field is assumed to be an incompressible fluid because its Mach number is significantly low. The equations of continuity and Navier-Stokes equations were adopted as the governing equations.

OpenFOAM-v1812 [7], an open-source computational fluid dynamics toolbox released under the GNU Gendral Public License (GPL), was adopted as the ftew field solver. Here, "pimpleFoam" was selected from the standard solvers. The PIMPLE nethod was the pressure-velocity coupling method. The finite volume metho the Navier-Stokes equations. Second-order accurate upwind schemes were adopted to evaluate the advection and viscous Euler implicit method was applied for time integration.

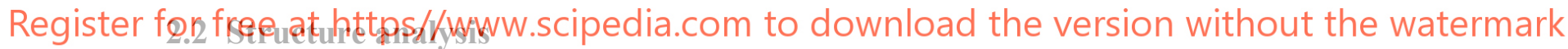

CalculiX-v2.15 [8], an open-source structural finite element solver released under the GPL, was adopted for the structural analysis.

The governing equations for the dynamic analysis were formulated based on the principle of virtual work. The finite element method was applied for the discretization. A ten-node tetrahedral element was used as the discretization element. Direct integration dynamic analysis was selected as the analysis type, and the equations of motion were numerically integrated using the $\alpha$-method $[9,10]$. A consistent mass matrix was adopted for the discrete mass system.

\subsection{Coupling method}

A partitioned coupling tool, preCICE [5], was used to establish intercommunication between OpenFOAM and CalculiX and perform FSI analysis.

PreCICE was developed by the Technical University of Munich and the University of Stuttgart. It is a collection of libraries and tools used for integrating coupled analyses. This coupling tool can effectively link various analytical tools.

A conventional serial staggered scheme was adopted as the coupling scheme. Force 
information was passed from the fluid solver to the structure solver, while displacement information was passed from the structure solver to the fluid solver. The scheme adopted involves a one-time exchange of physical quantities at the coupled boundary.

Nearest projection mapping was adopted as the data mapping method. With this method, physical information can be mapped from node to node by interpolation, even when the grids do not match the coupled boundary.

\section{COMPUTATIONAL DOMAIN AND CONDITIONS}

\subsection{Fluid analysis}

The parafoil used in the wind tunnel possessed an arch wing with chord and span lengths of 0.467 [m] and $1.4[\mathrm{~m}]$, respectively. The interior of the wing was divided into five cells using rigid ribs made of balsa wood. However, to validate the proposed FSI analysis model, a straight wing was adopted as the analytical object. The airfoil model is LA2573 A [11], which was also used in the JAXA low-speed wind tunnel test earlier described.

The airfoil was set in the computational domain at a $0^{\circ}$ angle of attack of. The surface of the airfoil was laminated the computational grids of 10 thin prism-shaped layers to resolve the boundary layer. The kinematic viscosity and air density of the fluid were set to $1.487 \times 10^{-5}$ $\left[\mathrm{m}^{2} / \mathrm{s}\right]$ and $1.2\left[\mathrm{~kg} / \mathrm{m}^{3}\right]$, respectively. Figure 2 illustrates the computational domain and the grids, and the calculation conditions are presented in Table 1.
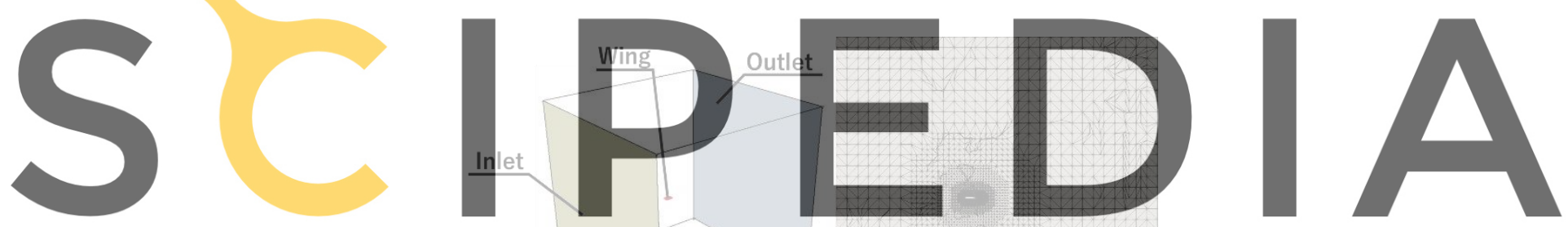

Register for free at https//www.scipedia.com to download the version without the watermark

Figure 2: Computational domain and grids

Table 1: Calculation conditions

\begin{tabular}{|c|c|}
\hline Calculation domain & $10 \times 10 \times 10\left[\mathrm{~m}^{3}\right]$ \\
\hline Dynamic viscosity & $1.487 \times 10^{-5}\left[\mathrm{~m}^{2} / \mathrm{s}\right]$ \\
\hline Density & $1.2\left[\mathrm{~kg} / \mathrm{m}^{3}\right]$ \\
\hline Wing & movingWallVelocity \\
\hline Inlet & \\
\hline Outlet & $\mathrm{du} / \mathrm{dx}=0$ \\
\hline Reynolds number & $4.1 \times 10^{5}$ \\
\hline
\end{tabular}




\subsection{Structure analysis}

Unlike conventional parafoils, the proposed FEM model is defined as a solid to validate the analysis method and conduct stable numerical analysis. This is to prevent divergence in the calculation owing to the significant strain in the grid triggered by the large displacement of the shell structure. The wing of the parafoil was made of polyimide. The material properties were determined by referring to the catalog specifications of polyimide and regarded as an isotropic material. As illustrated in Fig. 3, the analysis was conducted with the wing tip as the constraint condition. Figure 3 illustrates the FEM analysis model, and Table 2 presents the physical properties of the wing components.
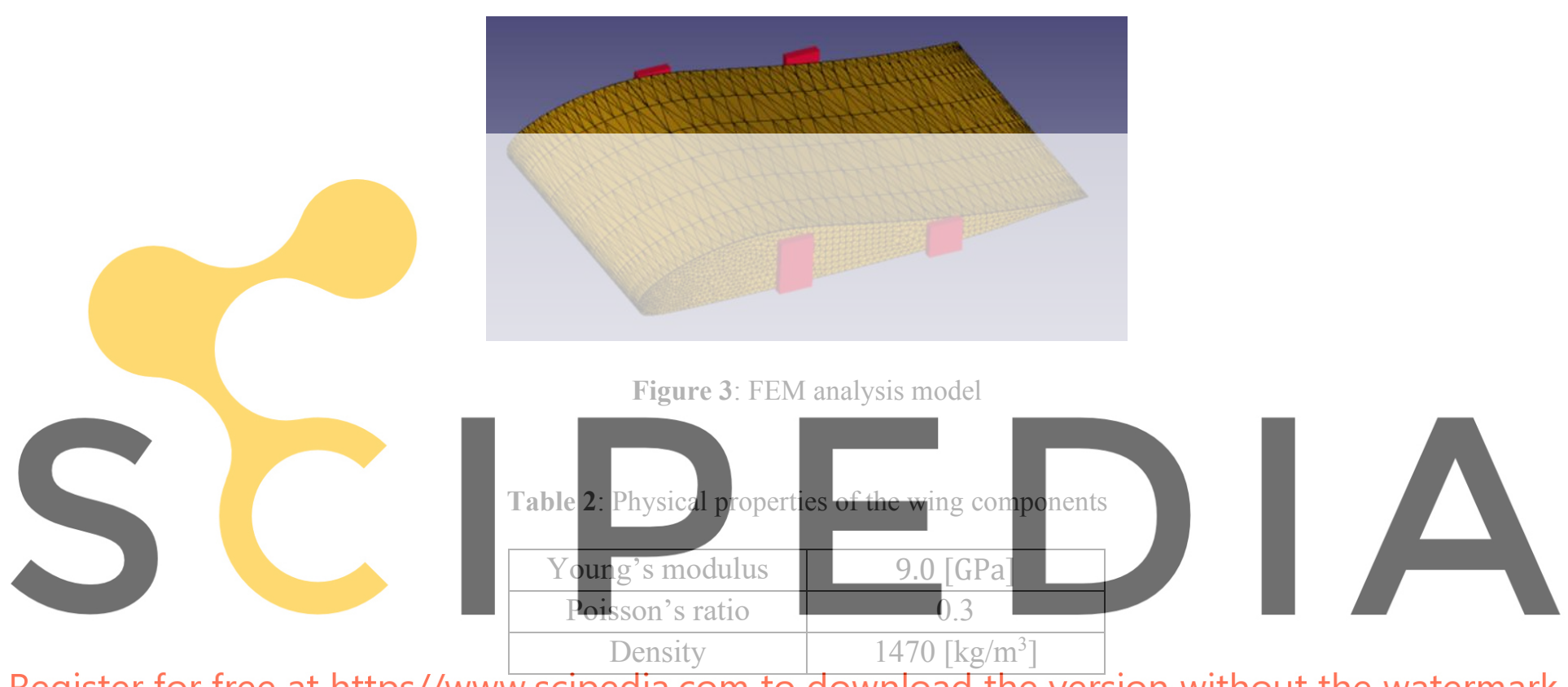

\section{Register for free at https//www.scipedia.com to download the version without the watermark}

\section{RESULTS AND DISCUSSIONS}

First, we performed structure- and fluid-only analyses using CalculiX and OpenFOAM, respectively. Subsequently, we conducted fluid-structure interaction analysis. We then investigated the characteristics of the coupled analysis based on the results obtained from the structure and fluid analyses. The lift coefficient (CL), pitching moment coefficient (CM), and drag coefficient (CD) are defined by the following equations:

$$
\begin{gathered}
C L=\frac{L}{\frac{1}{2} \rho S U^{2}} \\
C M=\frac{M}{\frac{1}{2} \rho S U^{2} c} \\
C D=\frac{D}{\frac{1}{2} \rho S U^{2}}
\end{gathered}
$$


where L, M, and D are the lift force, pitching moment, and drag force, respectively, while $\rho, S$, $U$, and $c$ are the density, representative area, representative velocity, and representative length, respectively.

\subsection{Fluid-only analysis}

Figure 4 presents the history of aerodynamic coefficients and fast Fourier transform (FFT) analysis results obtained for the lift coefficient in the fluid-only analysis. The lift, drag, and pitching moment coefficients are shown depicted as $\mathrm{CL}, \mathrm{CD}$, and $\mathrm{CM}$, respectively. To eliminate the initial value, the applicable range for the FFT analysis is $0.0051-0.5000$ [s].

Because the airfoil surface is a rigid body in the fluid-only analysis, deformations do not occur, and the aerodynamic coefficient is constant. The FFT analysis for CL indicated that the characteristic frequencies of the fluid origin are distributed below 100 [Hz].

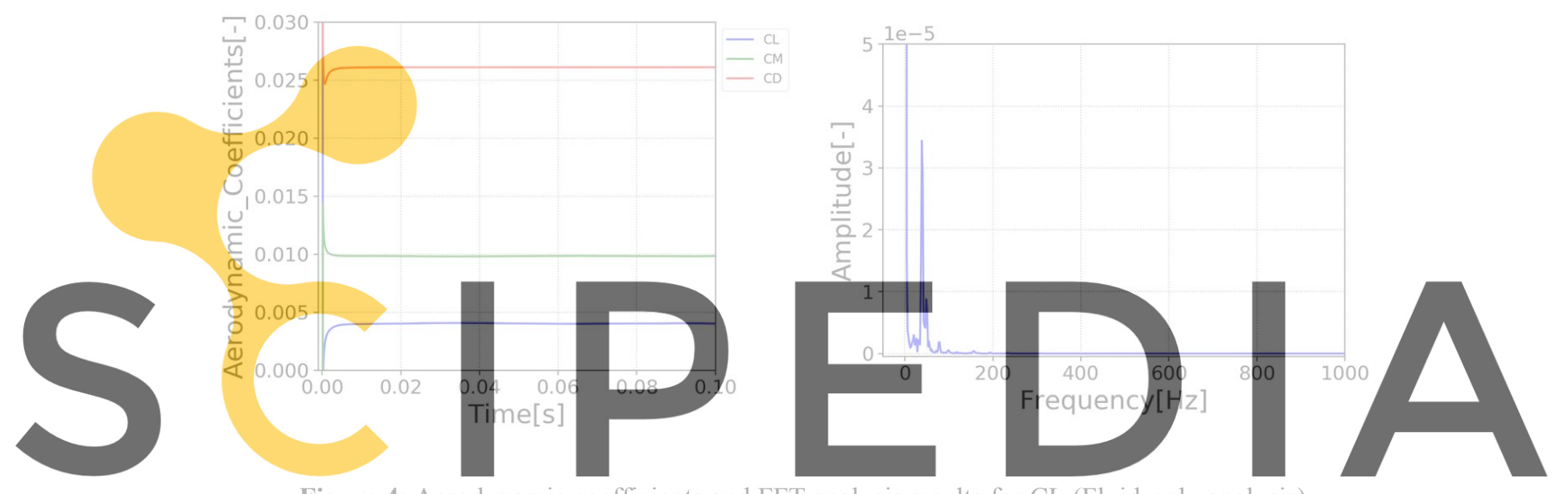

Figure 4: Aerodynamic coefficients and FFT analysis results for CL (Fluid-only analysis)

\section{Register forf free at https/dwww.scipedia.com to download the version without the watermark}

The eigenfrequency analysis of the solid model was performed using CalculiX, as illustrated in Figure 3. Table 3 presents the frequencies from Modes 1 to 5, and Figure 5 presents the absolute value of the displacement in the eigenmode 1.

It was inferred that Mode 1 tends to oscillate near the trailing edge of the wing.

Table 3: Eigenfrequencies of the proposed analysis model

\begin{tabular}{|c|c|}
\hline Eigenmode & {$[\mathbf{H z}]$} \\
\hline $\boldsymbol{f}_{\mathbf{1}}$ & 793.48 \\
\hline $\boldsymbol{f}_{\mathbf{2}}$ & 1391.05 \\
\hline $\boldsymbol{f}_{\mathbf{3}}$ & 1483.42 \\
\hline $\boldsymbol{f}_{\mathbf{4}}$ & 1574.93 \\
\hline $\boldsymbol{f}_{\mathbf{5}}$ & 1965.54 \\
\hline
\end{tabular}




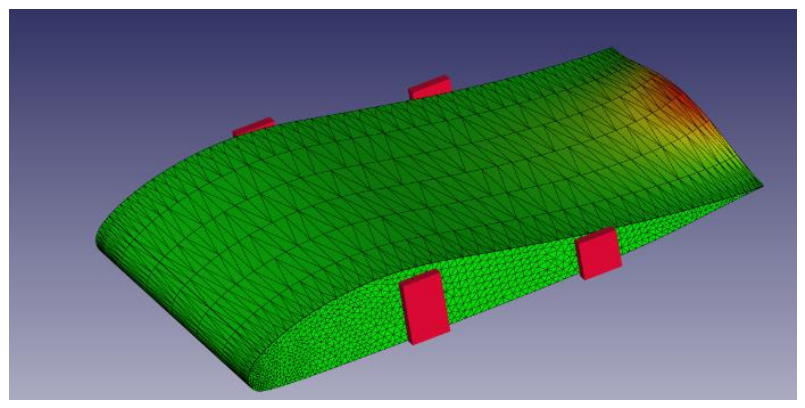

Figure 5: Absolute value of displacement (eigenmode: $\boldsymbol{f}_{\mathbf{1}}$ )

\subsection{Fluid structure interaction analysis}

Figure 6 illustrates the distribution of the absolute displacement values, while Figure 7 presents the displacement time history of the upper surface node of the wing and FFT analysis results for the displacement in the y-direction. In addition, Fig. 8 presents the aerodynamic coefficients and FFT analysis results for the lift coefficient in the FSI analysis. Similar to the fluid-only analysis, FFT analysis is performed on the data obtained, which eliminates the initial value variation.

Figure 6 indicates that this FSI analysis model can capture the structure's oscillation under fluid force, although the

this distribution,

According to the time

direction accounts for

of the $x-, y-$, and $z$-axes.

direction. Because the frequency with the

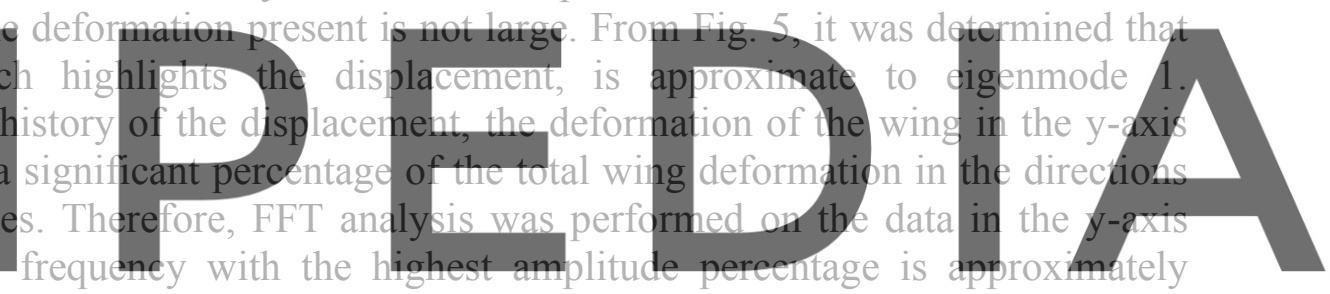

$793[\mathrm{~Hz}]$, the structure receives fluid force and starts to oscillate at its own eigenfrequency in

folrofiree at https//www.scipedia.com to download the version without the watermark

The influence of wing deformation on aerodynamic forces was evaluated. Compared with

the results of the fluid-only analysis, it is determined that the aerodynamic coefficients obtained by the FSI analysis exhibit high-frequency components. To investigate the reason for the oscillation, FFT analysis was performed for the CL. Frequencies lower than $100[\mathrm{~Hz}]$ were derived from the fluid by comparing them with those of the fluid-only analysis. Similarly, a frequency of approximately $793[\mathrm{~Hz}]$ was derived from the structure by comparison with natural frequency analysis.

Compared with the results of the fluid-only analysis, it was inferred that the wing surface oscillates in the FSI analysis, which is caused by the structure-derived high-frequency component that appears in the aerodynamic force. However, in accordance with the results of the aerodynamic force and FFT analysis, the high-frequency component gradually decreased. Finally, the aerodynamic coefficient was the same as that obtained from the fluid-only analysis. In this analysis, the aerodynamic force was not affected by the wing surface oscillation alone. One of the reasons for this phenomenon is that the geometry of the wing model differs from that of an actual aircraft. In this study, the material properties of the structure are considered the catalog specifications of the polyimide used in the actual parafoil of the wind tunnel test earlier described. However, for simplicity, a solid model was adopted. 
This model is different from the actual shape of the parafoil and has no cavity space inside the wing. Based on these factors, it was inferred that the stiffness of the proposed model is higher than that of the actual parafoil; therefore, its wing surface is not easily deformed.

Time: $0.001000(s)$
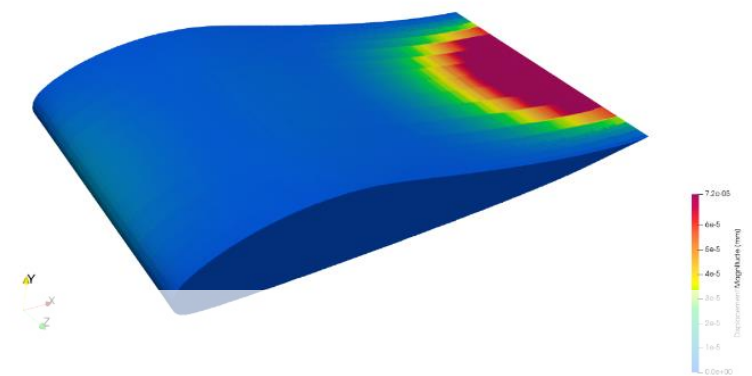

Figure 6: Absolute value of displacement (FSI analysis)
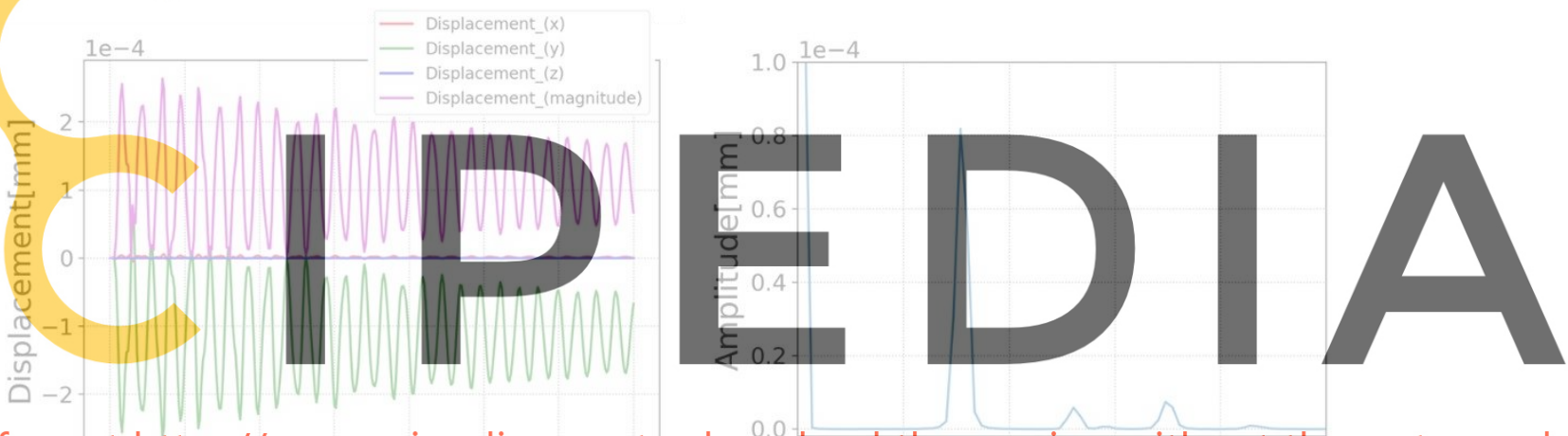

Register for free at https//www.Scipedia.com to downdoadsthe versionowithout theo watermark
Frequency[Hz] Time[s]

Figure 7: Time history of wing upper surface node displacement and FFT analysis results for the displacement in y-direction
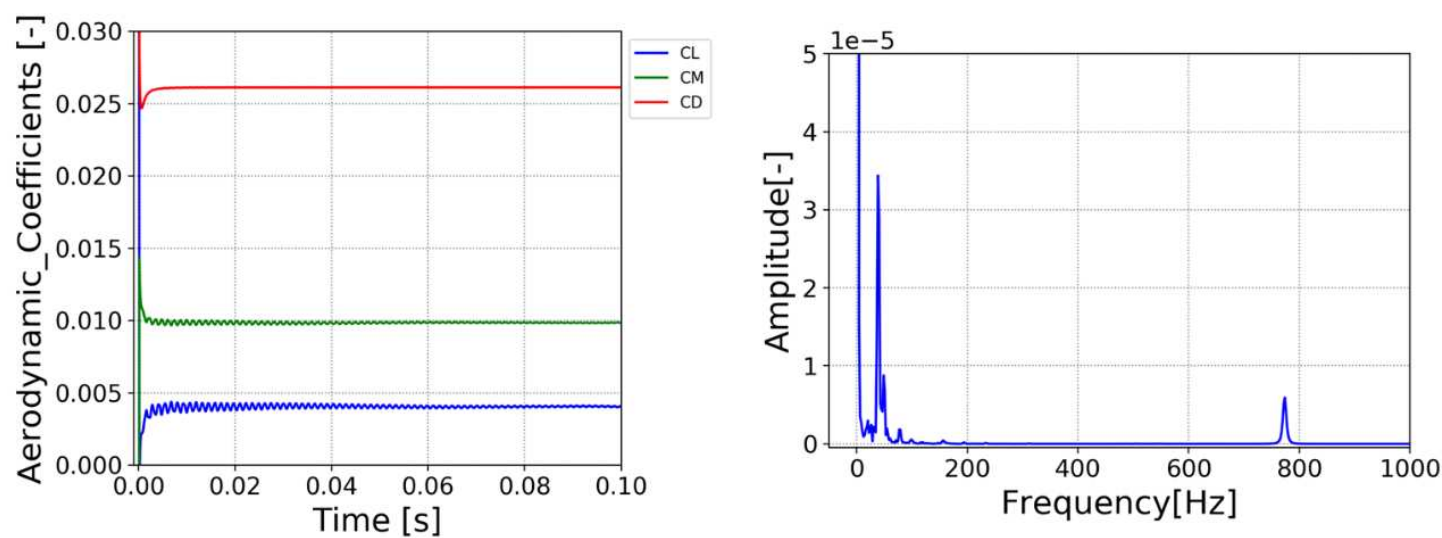

Figure 8: Aerodynamic coefficients and FFT analysis of CL (FSI analysis) 


\section{CONCLUSIONS}

To elucidate the aerodynamic behavior of a partially sealed parafoil for Mars exploration, an FSI analysis model was developed considering the effect of wing surface deformation. We concluded that the influence of wing deformation on aerodynamic forces is negligible because the stiffness obtained from the model was higher than that of the actual parafoil, which suppressed deformations. Further studies should be conducted to develop analysis models that are closely similar to actual machine shapes.

\section{ACKNOWLEDGEMENTS}

This work was supported by JSPS KAKENHI (Grant Number 20H02360), and by the "Joint Usage/Research Center for Interdisciplinary Large-scale Information Infrastructures" and "High Performance Computing Infrastructure" in Japan (jh200035)

We would like to thank Editage (www.editage.com) for English language editing.

\section{REFERENCES}

[1]Kirin. Tanishige, et al., 19-20 December, 2011 "Wind tunnel test results of a sealed parafoil for Mars exploration applications". in, Institute of Space and Astronautical Science, Japan Aerospace Exploration Agency (JAXA)(ISAS)), Sagamihara, Kanagawa Japan, Symposium on Elight Mechanics and Astrodynamics 2011 Japanese "Kasei

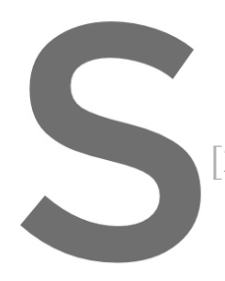
Tansa Heno Ouyo Tsuite").

[2]Hisashi. Nagano, et on Flight Mechanics and Astrodynamic Hudou Shiken"
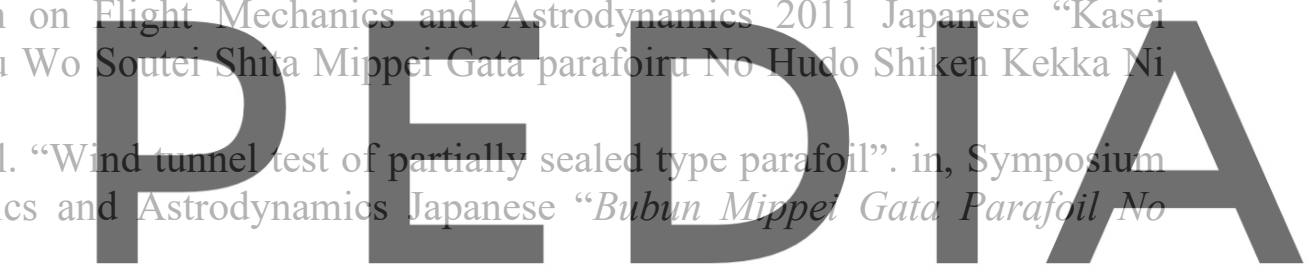

[3]Kunihiro. Ishida, et al., 2020 "Fluid-structure interaction analysis for Martian exploration

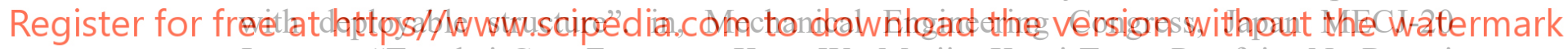
Japanese "Tennkai Gata Zyuunann Kozo Wo Motiita Kasei Tansa Parafoiru No Ryutai Kozo rensei kaiseki").

[4]Takahiro. Moriyoshi, et al. "Comparison of flight test and wind tunnel test on parafoil-type vehicle". in, 62nd Space Sciences and Technology Conference Japanese "Parafoiru Gata Hisyoutai Ni Okeru Huraito Shiken To Hudou Shiken No Hikaku").

[5]Hans J. Bungartz, Florian. Lindner, Bernhard. Gatzhammer, Miriam. Mehl, Klaudius. Scheufele, Alexander. Shukaev, Benjamin. Uekermann "preCICE - A fully parallel Library for Multi-Physics Surface Coupling". in Computers and Fluids, 141(2016), 250.

[6]Fraunhofer Institute for Algorithms and Scientific Computing, MpCCI, Germany (SCAI), Sankt Augustin 4.4.1-1 Documentation, 2015. http://www.mpcci.de/fileadmin/mpcci/download/MpCCI-4.4.1/doc/pdf/MpCCIdoc.pdf.

[7]'OpenFOAM project webpages’ Webpage, 2011. URL http://www.openfoam.org, accessed 20220-07-09.

[8]G. Dhondt, K. Witting 'CalculiX: A Free Software Three-Dimensional Structural Finite Element Program’ Webpage, 1998. URL http://www.calculix.de/.

[9]I. Miranda, R.M. Ferencz, T.J.R. Hughes "An improved implicit-explicit time integration method for structural dynamics" Earthquake Engineering and Structural Dynamics, 
18(1989), 643.

[10]G. Dhondt, 2004, “The Finite Element Method for Three-Dimensional Thermomechanical Applications", John Wiley \& Sons.

[11]'Airfoil Tools' Webpage, 2020. URL http://airfoiltools.com/. 